\section{Lesiones focales hepáticas benignas: un hallazgo frecuente a la tomografía computada}

\author{
GLORIA HORTA $^{1, \mathrm{a}}$, MARCELO LÓPEZ ${ }^{2, \mathrm{~b}}$, ANDRÉS DOTTE $^{2, \mathrm{~b}}$, \\ JORGE CORDERO $^{1, a}$, CATERINA CHESTA $^{\mathrm{c}}$, ARIEL CASTRO $^{\mathrm{d}}$, \\ PATRICIO PALAVECINO ${ }^{2}$, JAIME PONIACHIK ${ }^{1}$
}

\section{Benign focal liver lesions detected by computed tomography. Review of 1,184 examinations}

\begin{abstract}
Background: Multidetector computed tomography (MDCT) of the abdomen, with use of contrast medium, is able to detect and differentiate most focal liver lesions. Aim: To determine the prevalence and features of benign focal liver lesions (BFLL) detected by abdominal MDCT. Patients and Methods: We reviewed the reports of contrast abdominal MDCT performed to outpatients between August 2011 and July 2012. Clinical data of examined patients and imaging findings in terms of description of the hepatic parenchyma and the presence of BFLL, were recorded. Results: Data from 1,184 studies were analyzed. Of these, 461 studies (38.4\%) reported BFLL. The most prevalent lesions were simple cysts in $290 \mathrm{stu}$ dies (24\%) and hemangiomas in 61 studies (5.1\%), granuloma-calcification in $39(3.2 \%)$, focal nodular hyperplasia in 19(1.6\%) and one adenoma. If patients with known causes of liver disease were excluded, the prevalence of BFLL did not change substantially (lesions were found in 396 (37.5\%) patients). Compared with livers with signs of damage, normal livers had more cystic lesions (27 and $16.2 \%$ respectively, $p=0.014$ ) and hemangiomas (5.3 and $1.1 \%$ respectively, $p=0.043)$. Conclusions: BFLL are very common findings in MDCT studies. Most of these lesions are simple cysts and hemangiomas.
\end{abstract}

(Rev Med Chile 2015; 143: 197-202)

Key words: Cysts; Liver neoplasms; Tomography, X-Ray, computed.

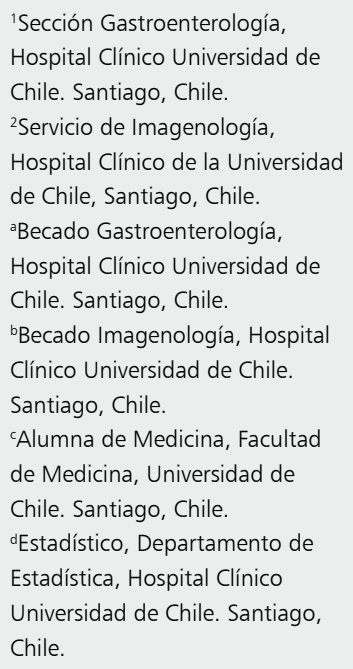

L

os estudios de imágenes, cada vez más requeridos para el estudio de diversas patologías, junto con la significativa mejoría en técnicas y equipos ha llevado a un importante aumento en la detección y diagnóstico de lesiones hepáticas focales. Cuando estas lesiones son encontradas en un paciente asintomático y sin antecedentes previos que lo describan, constituyen un hallazgo incidental o incidentaloma ${ }^{1-3}$. El hallazgo de estas lesiones varía entre 12 a 52\% en distintas series, según la población estudiada y el método diagnóstico utilizado ${ }^{4,5}$. La mayoría de estas lesiones, resultan ser benignas; entre las más frecuentes se describen el hemangioma, el quiste simple y la hiperplasia nodular focal ${ }^{6}$. La tomografía computada multidetector (TCMD), gracias a la rapidez de barridos, es capaz captar imágenes tanto en la fase arterial y venosa de la inyección de medio de contraste y realiza cortes de alta resolución de 1-2 $\mathrm{mm}$ de espesor en diferentes planos, todo lo cual es de suma utilidad al momento de caracterizar las lesiones hepáticas ${ }^{7}$. Estudios han demostrado un muy buen rendimiento de este examen para la detección y caracterización de los distintos tipos de lesiones hepáticas, con resultados comparables a los encontrados por resonancia magnética $(\mathrm{RM})^{8}$, excepto en lesiones asociadas a daño hepático o lesiones muy pequeñas en las que esta última 
logra una mejor caracterización ${ }^{9,10}$. Cuando las características de las imágenes ya sea por TCMD o RM, son categóricas, no se requiere de ningún otro examen de confirmación como por ejemplo anatomía patológica, ya que la correlación imagen-histología es tan alta que la biopsia se reserva sólo a casos muy puntuales. Los datos de prevalencia de lesiones focales hepáticas benignas (LFHB) conocidos hasta ahora provienen sólo de datos extranjeros y no existen estudios en población chilena o latinoamericana que entreguen esta información. El propósito de este estudio es conocer la prevalencia y caracterizar los tipos de LFHB más frecuentes en nuestro medio.

\section{Pacientes y Métodos}

Se realizó un estudio retrospectivo, descriptivo, en que se revisaron las TCMD de abdomen con contraste realizadas en forma ambulatoria entre octubre de 2011 y julio de 2012, solicitados por múltiples etiologías al Servicio de Radiología del Hospital Clínico de la Universidad de Chile. Todos los estudios incluían un topograma sin contraste y otro en fase venosa portal. Además se incluía fase arterial en aquellos estudios solicitados por neoplasia-metástasis, isquemia o enfermedad vascular, y en pacientes con antecedentes de hepatopatía o lesión focal hepática en estudio. En estas últimas se agregaba además la fase venosa tardía o de equilibrio para mejor caracterización. Se revisó el informe y en forma prospectiva se revisaron las imágenes, discutiendo con un segundo radiólogo observador aquellas imágenes que no estuvieren claramente descritas en el informe. Se obtuvieron datos de edad, sexo, antecedente clínico por el cual se solicitaba el examen, y se registraron los hallazgos tomográficos. El hígado era descrito como normal, esteatósico o con signos de daño hepático crónico. La determinación de hígado graso o esteatósico se hizo en base a la hipoatenuación o disminución de densidad difusa típica que presenta el hígado con infiltración grasa, definida tomográficamente como una densidad del parénquima hepático menor a 40 Unidades Hounsfield (UH) o menos de $10 \mathrm{UH}$ en comparación con el bazo.

Los signos de daño hepático crónico a la tomografía consistían en la disminución del tamaño hepático con prominencia relativa de los segmen- tos laterales del lóbulo izquierdo y del caudado, estructura hepática heterogénea y/o superficie nodular-lobulada.

Las lesiones focales hepáticas encontradas fueron clasificadas por tipo, número de lesiones, tamaño de la lesión mayor, ubicación, y coexistencia de más de un tipo de lesión en un mismo paciente.

Las variables según su naturaleza se presentaron en promedio y desviación estándar, percentiles 25,50 y 75 en caso de las variables cuantitativas y frecuencias expresadas en porcentaje para variables cualitativas. El análisis estadístico se realizó con la prueba de comparación de proporciones y las pruebas de chi-cuadrado y test de Fisher. Se consideró estadísticamente significativo $\mathrm{p}<0,05$.

\section{Resultados}

Se analizó la TCMD de abdomen con contraste de 1.184 pacientes, 498 (42\%) de sexo masculino con un promedio de edad de $57 \pm 16$ años (15-96 años). De éstos, 526 (44\%) pacientes presentaron alguna lesion hepática focal (benigna o maligna), y en $76(6,4 \%)$ de los cuales coexistía más de un tipo de lesión. Se encontraron 523 LFHB en 461 $(38,4 \%)$ pacientes.

Las LFHB encontradas fueron: quiste simple en 290 (24\%), hemangioma en $61(5,1 \%)$, granuloma-calcificación en 39 (3,2\%), hiperplasia nodular focal en $19(1,6 \%)$ y un caso de adenoma. Hubo 113 (9,5\%) lesiones que no fueron definidas categóricamente y sólo fueron catalogadas por su característica principal en hipervascular, hipovascular, hiperdensa, hipodensa o indeterminada si no clasificaba en ninguna de las anteriores.

El promedio de edad de los pacientes con quiste simple y hemangioma fue de $61 \pm 13$ años, con granuloma-calcificación fue de $67 \pm 11$ años y con lesiones no determinadas de $57 \pm 14$ años. Los pacientes con hiperplasia nodular focal presentaron un promedio de edad de $44 \pm 18$ años y se presentaron significativamente más frecuentemente en mujeres que en hombres $(2,1 \%$ vs $0,8 \%$ $\mathrm{p}<0,01)$. El resto de las lesiones no presentaron diferencias significativas en la distribución por sexo (Tabla 1).

El motivo por el cual se solicitó el examen, registrado en $1.004(85 \%)$ pacientes, resultó más frecuentemente relacionado con estudio de enfer- 
Hallazgos tomográficos en lesiones hepáticas benignas - G. Horta et al

Tabla 1. Distribución de lesiones según sexo y edad

\begin{tabular}{|c|c|c|c|}
\hline & \multicolumn{2}{|c|}{ Sexo } & \multirow[t]{2}{*}{ Edad } \\
\hline & n hombres (\%) & n mujeres (\%) & \\
\hline Quiste simple & $112(22)$ & $178(26)$ & $61 \pm 13$ \\
\hline Hemangioma & 17 (3) & 44 (6) & $61 \pm 14$ \\
\hline Granuloma-calcificación & 18 (3) & $21 \quad(4)$ & $67 \pm 11$ \\
\hline Hiperplasia nodular focal & $4(2)$ & $15(0,8)$ & $44 \pm 18$ \\
\hline Adenoma & $1 \quad(0,2)$ & 0 & 51 \\
\hline No determinada & $53(11)$ & $60 \quad(9)$ & $57 \pm 14$ \\
\hline Total & 205 & 318 & \\
\hline
\end{tabular}

Tabla 2. LFHB encontradas; prevalencia grupo total vs prevalencia grupo con exclusión de pacientes con antecedente de lesión y/o daño hepático crónico

\begin{tabular}{|lccc|}
\hline Lesión & $\begin{array}{c}\text { Grupo total } \\
\text { n: 1.184 (\%) }\end{array}$ & $\begin{array}{c}\text { Subgrupo sin antecedentes de lesión/ } \\
\text { daño hepático n: 1.055 (\%) }\end{array}$ & p-value \\
Quiste simple & $290(24,4)$ & $264(25)$ & 0,371 \\
Hemangioma & $61(5,1)$ & $48(4,5)$ & 0,254 \\
Granuloma-calcificación & $39(3,2)$ & $38(3,6)$ & 0,3 \\
Hiperplasia nodular focal & $19(1,6)$ & $11(1)$ & 0,107 \\
Adenoma & $1(0,08)$ & $1(0,09)$ & 0,398 \\
No determinada & $113(9,5)$ & $93(8,8)$ & 0,28 \\
Total & 602 & 502 & \\
\hline
\end{tabular}

medades neoplásicas, ya sea sospecha diagnóstica, etapificación o seguimiento en 432 (36\%), seguido del estudio de dolor abdomino-pelviano en 224 (23\%) y misceláneas en 253 (21\%) dentro de las que se incluían baja de peso en estudio, alteraciones del tránsito intestinal, cuadros inflamatorios intraabdominales, trauma y otros. Sólo en 94 (8\%) pacientes existía antecedente de patología hepática conocida (daño hepático o lesiones focales).

Con respecto a las características tomográficas del hígado, 951 (80\%) fueron catalogados como normales, seguido de aquellos con signos de hipoatenuación sugerente de esteatosis en 147 $(12,3 \%)$ y de aquellos con signos de daño hepático crónico en 86 (7,3\%). Excluyendo los casos de enfermedad o lesión hepática conocida y/o signos de daño hepático crónico (total 1.055 pacientes), se encontraron 455 LFHB en 396 (37,5\%) pacientes, manteniendo similar prevalencia que el total general $(38,5 \%)$ (Tabla 2$)$. Por otra parte, las lesiones quísticas fueron más frecuentemente encontradas en hígado normal que en hígado con signos de daño hepático crónico ( $27 \%$ vs $16,2 \% \mathrm{p}=0,014)$ o esteatosis $(27 \%$ vs $12,9 \% \mathrm{p}<0,01)$ y resultó llamativa la ausencia de granuloma-calcificación y el significativo menor hallazgo de hemangiomas en los hígados con signos de daño hepático crónico en comparación con los hígados normales $(3,7 \%$ vs $0 \% \mathrm{p}=0,03$ y $5,3 \%$ vs $1,1 \% \mathrm{p}=0,043$ respectivamente). También es importante destacar que las alteraciones del parénquima hepático, en especial la esteatosis, estuvieron relacionadas con un mayor número de lesiones de carácter no determinado en comparación con los hígados de aspecto normal $(8,1 \%$ vs $0,9 \% \mathrm{p}<0,01)$ (Tabla 3$)$.

Las lesiones se presentaban como únicas (46\%) o múltiples (54\%). Todos los tipos de lesiones podían presentarse como lesión única o múltiple, pero encontramos algunas con mayor tendencia a presentarse como lesión única como el hemangio- 
Tabla 3. Distribución de LFHB en relación a las características del hígado, valor de p: comparación con hígado normal

\begin{tabular}{|lccccc|}
\hline Lesión & $\begin{array}{c}\text { Hígado normal } \\
\text { n: 951 (\%) }\end{array}$ & $\begin{array}{c}\text { Esteatosis } \\
\text { n: 147 (\%) }\end{array}$ & p-value & $\begin{array}{c}\text { Daño hepático } \\
\text { crónico } \\
\text { n: 86 (\%) }\end{array}$ & p-value \\
\hline Quiste simple & $257(27,0)$ & $19(12,9)$ & $<0,01$ & $14(16,2)$ & 0,01 \\
\hline Hemangioma & $51(5,3)$ & $9(6,1)$ & 0,34 & $1(1,1)$ & 0,04 \\
\hline Granuloma-calcificación & $36(3,7)$ & $3(2,0)$ & 0,15 & $0(0,0)$ & 0,03 \\
Hiperplasia nodular focal & $16(1,6)$ & $2(1,3)$ & 0,39 & $1(1,1)$ & 0,36 \\
Adenoma & $1(0,1)$ & $0(0,0)$ & 0,35 & $0(0,0)$ & 0,38 \\
No determinada & $90(0,9)$ & $12(8,1)$ & $<0,01$ & $11(1,2)$ & 0,39 \\
Total & 451 & 45 & & 27 & \\
\hline
\end{tabular}

Tabla 4. Características de LFHB

\begin{tabular}{|lccccccccccc|}
\hline & \multicolumn{3}{c}{$\begin{array}{c}\text { Presentación en número* } \\
\end{array}$} & U (\%) & M (\%) & NR (\%) & LD (\%) & LI (\%) & O (\%) & NR (\%) & $\begin{array}{c}\text { Tamañcación** lesión } \\
\text { mayor } \\
\text { Mediana (mm) }\end{array}$ \\
Quiste simple & $153(53)$ & 131 & $(45)$ & $6(2)$ & 118 & $(41)$ & $100(34)$ & 27 & $(9)$ & $45(16)$ & 10 \\
Hemangioma & $37(60)$ & 21 & $(34)$ & $3(6)$ & 35 & $(58)$ & $16(26)$ & 2 & $(3)$ & $8(13)$ & 8 \\
Granuloma-calcficación & $24(61)$ & 12 & $(31)$ & $3(8)$ & 22 & $(56)$ & $13(33)$ & $3(8)$ & 1 & $(3)$ & 10 \\
Hiperplasia nodular focal & $14(74)$ & 5 & $(26)$ & 0 & 6 & $(32)$ & $9(47)$ & $2(16)$ & 1 & $(5)$ & 9,5 \\
Adenoma & 0 & $1(100)$ & 0 & $1(100)$ & 0 & 0 & 0 & 15 \\
No determinada & $64(56)$ & $44(39)$ & $5(5)$ & $56(50)$ & $35(33)$ & $6(5)$ & $16(12)$ & 9,5 \\
\hline
\end{tabular}

*U: única, M: múltiple, NR: no registrado. **LD: lóbulo derecho, LI: lóbulo izquierdo, O: otros (caudado, bilateral, difuso), NR: no registrado.

ma $(60 \%)$, la hiperplasia nodular focal $(73 \%)$ y el granuloma-calcificación $(60 \%)$. Como gran parte de las lesiones se presentaban como múltiples sólo fue registrado el tamaño de la lesión mayor. Se obtuvo la mediana del tamaño de lesión mayor, el que para la mayoría de las lesiones fue de 10 mm (Tabla 4).

Hubo una tendencia a encontrar las lesiones más frecuentemente en segmentos del lóbulo derecho, exceptuado la hiperplasia nodular focal que se presentó más frecuentemente en segmentos del lóbulo izquierdo (Tabla 4).

\section{Discusión}

Encontramos una alta prevalencia $(38,4 \%)$ de lesiones hepáticas focales benignas como hallazgo a la TCMD. Incluso cuando descartamos los casos en cuyo antecedente existía conocimiento de alguna lesión o enfermedad hepática o signos de daño hepático a la TCMD, es decir, verdaderos incidentalomas, se mantiene este hallazgo $(37,5 \%)$. Sin embargo, nos estamos refiriendo a unos sujetos con alguna manifestación clínica en que la solicitud del examen va acompañado de una sospecha clínica que lo justifique.

En este estudio un alto porcentaje de pacientes tenía antecedentes de alguna neoplasia en estudio, hecho que se entiende por la alta complejidad y referente de derivación de nuestro centro. Aún así nuestros hallazgos son comparables a los descritos en otras series ${ }^{11} \mathrm{y}$ en cuanto a las LFHB parecen ser igualmente frecuentes en pacientes con o sin 
Hallazgos tomográficos en lesiones hepáticas benignas - G. Horta et al

enfermedades neoplásicas ${ }^{6}$. Llama la atención la distribución de las lesiones ya que en reportes europeos y norteamericanos se describe el hemangioma como la lesión hepática benigna más frecuente en la población general, con prevalencias que llegan hasta $7,4 \%{ }^{12-13}$. Esta cifra se asemeja a la prevalencia de hemangiomas encontrada en nuestro estudio $(5,1 \%)$, pero se encuentra muy por debajo del hallazgo de quiste simple encontrado en nuestra serie (24\%), casi uno de cada cuatro pacientes.

Respecto de esto, los primeros reportes publicados mostraban una prevalencia de quistes hepáticos no parasitarios en 0,5 a $2,5 \%$ de la población ${ }^{14}$. Sin embargo, estudios más recientes realizados con tecnología de TCMD muestran que los quistes hepáticos simples se presentan hasta en $18 \%$ de la población, lo que se acerca más a lo encontrado en nuestra serie y refuerza el hecho de que el aumento en la detección de lesiones hepáticas focales va en directa relación con las técnicas de imagen utilizadas.

La hiperplasia nodular focal que reportamos $(1,6 \%)$ es similar a la comunicada en otras series, incluyendo características como mayor prevalencia en mujeres en edad fértil ${ }^{15}$. Ahora bien, aunque en fase portal se pueden detectar la gran mayoría de las lesiones hipervasculares, la falta de la fase arterial precoz y venosa tardía en muchos exámenes puede haber afectado la caracterización de lesiones que, al no tener características típicas como la cicatriz central, hubieren quedado categorizadas como lesiones hipervasculares, es decir, no determinadas.

La relación entre las características del hígado, asociadas a los diferentes tipos de lesiones hepáticas, no se encuentra claramente establecida. En nuestra opinión, las lesiones de más alta prevalencia, lesiones quísticas y hemangiomas, fueron significativamente más frecuentes de encontrar en hígados normales que en aquellos alterados, con esteatosis o con signos de daño hepático crónico. Esto podría deberse a que las alteraciones del parénquima hepático pueden afectar la detección de algunas lesiones, en especial las más pequeñas. A este respecto cabe destacar que las alteraciones del parénquima hepático también afectaron la caracterización de las lesiones, ya que la mayoría de las lesiones no determinadas estuvieron asociadas a hígados con signos de esteatosis o daño hepático crónico. En estos casos está indicada la RM de hígado ya que permite la mejor caracterización de las lesiones ${ }^{9,10,17}$. No encontramos relación entre el tamaño de la lesión y su caracterización: lesiones que no lograron ser determinadas promediaban tamaños similares al resto de las LFHB.

En conclusión, las LFHB son un hallazgo frecuente en nuestro medio, siendo el quiste simple el más prevalente.

\section{Referencias}

1. Little J, Kenny J, Hollands M. Hepatic incidentaloma: a modern problem. World J Surg. 1990; 14 (4): 448-51.

2. Belli G, D'agostino A, Lannelli A, Marano I. Hepatic incidentaloma. Retrospective analysis of 35 cases. Int Surg. 1996; 81 (2): 144-8.

3. Kreft B, Pauleit D, Bachmann R, Conrad R, Kramer A, Schild H. Incidence and significance of small focal liver lesions in MRI. Rofo. 2001; 173 (5): 424-9.

4. Schwartz L, Gandras E, Colangelo S, Ercolani M, Panicek D. Prevalence and importance of small hepatic lesions found at CT in patients with cancer. Radiology, 1999; 210 (1): 71-4.

5. Karhunen P, A. Benign hepatic tumours and tumor like condition in man. J Clin Pathol. 1986; 39 (2): 183-8.

6. Jones E, Chezmar J, Nelson R, Bernardino M. The frecuency and significance of small $(<15 \mathrm{~mm})$ hepatic lesions detected by CT. AJR Am J Roetgenol 1992; 159: 535-9.

7. Hussain S, Semelka. Hepatic Imaging: Comparison of Modalities. Radiol Clin N Am 2005; 43: 929-47.

8. Torzilli G, Minagawa M, Takayama T, Inoue K, Hui A, Kubota K, et al. Accurate preoperative evaluation of liver mass lesions without fine needle biopsy. Hepatology 1999; 30 (4): 889.

9. Lee J. Computed body Tomography with MRI Correlation, Fourth Edition, Lippincott Williams and Wilkins, Vol 1, 2006.

10. Mortele K, Ros P. Cystic Focal Liver Lesions in the Adult: Differential CT and MR Imaging Features. Radiographics 2001; 21: 895-910.

11. Blumgart, Fong, Janargin. Hepatobiliary Cancer. American Cancer Society. 2001; 2: 31-42.

12. Gandolfi L, Leo P, Solmi L, Vitelli E, Verros G, Colecchia A. Natural history of hepatic haemangiomas: clinical and ultrasound study. Gut, 1999; 32: 677-80.

13. Motta G, Alonso E, Chirino R, González-Merino L. Caracterización de lesiones hepáticas focales con tomografía computada multidetector. Anales de Radiología México 2012; 1: 46-58. 
14. Gaines P, Sampson M. The prevalence and characterization of simple hepatic cysts by ultrasound examination. British J of Radiol, 1989; 62: 335-7.

15. Pons F, Llovet J. Approaching focal liver lesions. Rev Esp Enf Digestivas 2004; 96 (8): 567-77.

16. Bruix J, Sherman M, Llovet J, Beaugrand M, Lencioni R,
Burroughs AK, et al. Clinical management of hepatocellular carcinoma. Conclusions of the Barcelona-2000 EASL Conference. J Hepatol 2001; 35: 421-30.

17. Zamora E, Soffia P. Lesión focal hepática: ¿Tomografía Axial Computarizada o Resonancia Nuclear Magnética? Gastroenterol latinoam 2008; 19 (2): 101-5. 